PROCEEDINGS OF THE

AMERICAN MATHEMATICAL SOCIETY

Volume 29, Number 3, August 1971

\title{
ON THE ARITHMETIC NATURE OF DEFINITE INTEGRALS OF RATIONAL FUNCTIONS ${ }^{1}$
}

\section{A. J. VAN DER POORTEN}

Abstract. A. Baker's theorems on linear forms in the logarithms of algebraic numbers imply information on the arithmetic nature of definite integrals of rational functions. This paper pro vides a convenient formulation of these implied results.

1. In his monograph on transcendental numbers, C. Siegel $[3, p$. 97] observed that the theory of transcendental numbers at that time (1949) was as yet unable to determine the arithmetic nature of such integrals as

$$
\int_{0}^{1} \frac{d x}{1+x^{3}}=\frac{1}{3}\left(\log 2+\frac{\pi}{\sqrt{ } 3}\right) .
$$

More recently (1966), A. Baker mentions that his theorems on linear forms in the logarithms of algebraic numbers [1] imply the transcendence of the above integral. Baker's results have led to further "folk theorems" on the arithmetic nature of certain integrals but no general statement appears to have been formulated. In this paper we establish a convenient criterion for determining the arithmetic nature of such definite integrals of rational functions as we are able to deal with by virtue of currently established theorems.

Theorem. Let $P(z), Q(z)$ denote polynomials with algebraic coefficients and with no common polynomial factor. Denote by $\alpha_{1}, \alpha_{2}, \cdots$, $\alpha_{n}$ the distinct zeros of $Q(z)$ and by $r_{1}, r_{2}, \cdots, r_{n}$ the residues respectively at the poles of the rational function $P(z) / Q(z)$. Further let $\Gamma$ be some contour in the complex plane for which the definite integral

$$
\int_{\Gamma} \frac{P(z)}{Q(z)} d z
$$

exists, and suppose that $\Gamma$ is either closed, or has endpoints which are algebraic or infinite.

Received by the editors February 20, 1970 and, in revised form, October 5, 1970. AMS 1969 subject classifications. Primary 1032.

Key words and phrases. Linear form, algebraic number, transcendental number, rational function, definite integral, partial fraction expansion.

1 The writing of a note on this topic was suggested to me by Professor K. Mahler at the 10th Summer Research Institute of the Australian Mathematical Society, Hobart, 1970. 
Then the definite integral is algebraic if and only if

$$
\int_{\Gamma}\left(\sum_{k=1}^{n} \frac{r_{k}}{z-\alpha_{k}}\right) d z=0 .
$$

CoRollary. If further, $\operatorname{deg} P(z)<\operatorname{deg} Q(z)$ and the zeros of $Q(z)$ are distinct, then

$$
\int_{\Gamma} \frac{P(z)}{Q(z)} d z
$$

is either transcendental or zero.

In particular, in Siegel's example above, we see that $1+x^{3}$ has distinct zeros, whence, since the integral is obviously nonzero, it follows from the corollary that the integral is transcendental.

We shall show that the theorem and its corollary follow, modulo some straightforward argument, from a suitable corollary of Baker's theorem.

2. In the sequel let $\alpha_{1}, \alpha_{2}, \cdots, \alpha_{n} ; \beta_{1}, \cdots, \beta_{n}$ be algebraic numbers with all of $\alpha_{1}, \cdots, \alpha_{n}$ and at least one of $\beta_{1}, \cdots, \beta_{n}$ nonzero. Further let $\log \alpha_{1}, \cdots, \log \alpha_{n}$ be defined once and for all, and let $\alpha_{\mathbf{k}}^{\boldsymbol{\beta}_{\boldsymbol{k}}}$ as usual mean $\exp \left(\beta_{k} \log \alpha_{k}\right)$. Then we may formulate as much as we require of Baker's results in the following manner:

BAKeR's Lemma. Let $\log \alpha_{1}, \cdots, \log \alpha_{n}$ be linearly independent over $Q$, the field of rational numbers. Then

$$
\beta_{1} \log \alpha_{1}+\cdots+\beta_{n-1} \log \alpha_{n-1}+\beta_{n} \neq \log \alpha_{n}
$$

and even

$$
\alpha_{1}^{\beta_{1}} \cdots \alpha_{n-1}^{\beta_{n-1} e^{\beta_{n}}} \neq \alpha_{n} .
$$

K. Mahler [2] has shown that the following rather striking result is equivalent to the lemma:

If $\log \alpha_{1}, \cdots, \log \alpha_{n}$ are linearly independent over $Q$, the field of rational numbers, then $\log \alpha_{1}, \cdots, \log \alpha_{n}, 1$ are linearly independent over $A$, the field of all algebraic numbers.

Mahler [2] also mentions a number of corollaries on transcendence. Suppose $n \geqq 2$, then:

(a) if $\beta_{n} \neq 0$ then $\alpha_{1}^{\beta_{1}} \cdots \alpha_{n-1}^{\beta_{n-1}} e^{\beta_{n}}$ is transcendental;

(b) if $\log \alpha_{1}, \cdots, \log \alpha_{n-1}$ are linearly independent over $Q$, and at least one of $\beta_{1}, \cdots, \beta_{n-1}$ is irrational, then $\alpha_{1}^{\beta_{1}} \cdots \alpha_{n-1}^{\beta_{n-1}}$ is tran. scendental; 
(c) if $\log \alpha_{1} \neq 0, \cdots, \log \alpha_{n-1} \neq 0$, and $\beta_{1}, \cdots, \beta_{n-1}$ are linearly independent over $Q$, then $\alpha_{1}^{\beta_{1}} \cdots \alpha_{n-1}^{\beta_{n-1}}$ is transcendental.

In each case the assertions follow easily from Baker's Lemma by elementary linear algebra. The reader is referred to [2] for details.

The most useful formulation of Baker's result for our purposes is:

Lемма. If $\beta_{1} \log \alpha_{1}+\cdots+\beta_{n-1} \log \alpha_{n-1}$ is algebraic, then it is zero.

The lemma is clearly equivalent to Mahler's corollary (a) above; we describe its proof explicitly. Thus suppose that $\beta_{1} \log \alpha_{1}+\cdots+$ $\beta_{n-1} \log \alpha_{n-1}=-\beta_{n}$, with $\beta_{n} \neq 0$, algebraic. Then certainly not all of $\log \alpha_{1}, \cdots, \log \alpha_{n-1}$ are zero, and thus there are $m \geqq 1$ numbers among $\log \alpha_{1}, \cdots, \log \alpha_{n-1}$, say $\log \alpha_{1}, \cdots, \log \alpha_{m}$, linearly independent over $Q$, and rationals $r_{h k}, 1 \leqq h \leqq n-1 ; 1 \leqq k \leqq m$, not all zero, such that

$$
\log \alpha_{h}=\sum_{k=1}^{m} r_{h k} \log \alpha_{k}, \quad 1 \leqq h \leqq n-1 .
$$

Hence our supposition becomes

$$
\left(\sum_{h=1}^{n-1} \beta_{h} r_{h 1}\right) \log \alpha_{1}+\cdots+\left(\sum_{h=1}^{n-1} \beta_{h} r_{h m}\right) \log \alpha_{m}+\beta_{n}=0 .
$$

Since $\beta_{n} \neq 0$, not all the coefficients $B_{k}=\sum_{h=1}^{n-1} \beta_{h} r_{h k}$ can vanish, say $B_{m} \neq 0$. Then we obtain

$$
\left(\frac{-B_{1}}{B_{m}}\right) \log \alpha_{1}+\cdots+\left(\frac{-B_{m-1}}{B_{m}}\right) \log \alpha_{m-1}+\left(\frac{-B_{n}}{B_{m}}\right)=\log \alpha_{m},
$$

and since $\log \alpha_{1}, \cdots, \log \alpha_{m}$ are linearly independent over $Q$, we contradict Baker's Lemma.

3. Henceforth let $P(z), Q(z)$ denote polynomials with algebraic coefficients, and with no common polynomial factor. Suppose that the factorisation of $Q(z)$ into distinct linear factors is

$$
Q(z)=q_{0}\left(z-\alpha_{1}\right)^{\rho(1)} \cdots\left(z-\alpha_{n}\right)^{\rho(n)} .
$$

Then the rational function $P(z) / Q(z)$ has a partial fraction expansion of the form

(1) $\frac{P(z)}{Q(z)}=p(z)+\sum_{k=1}^{n}\left\{\frac{r_{k}}{z-\alpha_{k}}+\frac{r_{k, 2}}{\left(z-\alpha_{k}\right)^{2}}+\cdots+\frac{r_{k, p(k)}}{\left(z-\alpha_{k}\right) \rho(k)}\right\}$,

where we note that the polynomial $p(z)$ has algebraic coefficients, and that the numbers $\alpha_{k}, r_{k}, r_{k, h}$ are all algebraic. In particular $r_{k}$ is exactly the residue of $P(z) / Q(z)$ at the pole $\alpha_{k}, 1 \leqq k \leqq n$. 
As in the statement of the theorem, we take a contour which is either closed or has endpoints which are algebraic or infinite. We further assume that

$$
\int_{\Gamma} \frac{P(z)}{Q(z)} d z
$$

exists.

If $\Gamma$ contains $\infty$ then necessarily the existence of (2) implies that in (1) both $p(z)=0$ and $\sum r_{k}=0$. Thus in general the numbers

$$
\int_{\Gamma} p(z) d z \text { and } \int_{\Gamma} \frac{r_{k, h}}{\left(z-\alpha_{k}\right)^{h}} d z, \quad 2 \leqq h \leqq \rho(k), \quad 1 \leqq k \leqq n,
$$

can be immediately seen to be algebraic, whence the definite integral (2) is algebraic if and only if

$$
\int_{\Gamma}\left(\sum_{k=1}^{n} \frac{r_{k}}{z-\alpha_{k}}\right) d z
$$

is algebraic.

4. We assert that (3) is always a linear form over the algebraic numbers in the logarithms of algebraic numbers. Then by our lemma such an expression is either transcendental or zero, and we have the statement of the theorem.

Let $\gamma, \delta$ be two finite points on $\Gamma$ and denote by $\Gamma(\gamma, \delta)$ the curve from $\gamma$ to $\delta$ along $\Gamma$. Then

$$
\begin{aligned}
\int_{\Gamma(\gamma, \delta)}\left(\sum_{k=1}^{n} r_{k}\left(z-\alpha_{k}\right)^{-1}\right) d z & =\sum_{k=1}^{n} r_{k} \int_{\Gamma(\gamma, \delta)}\left(z-\alpha_{k}\right)^{-1} d z \\
& =\sum_{k=1}^{n} r_{k}\left\{\log \left(\gamma-\alpha_{k}\right)-\log \left(\delta-\alpha_{k}\right)\right\} \\
& =\log \left\{\prod_{k=1}^{n}\left(\frac{\gamma-\alpha_{k}}{\delta-\alpha_{k}}\right)^{r k}\right\}+2 \pi i m(\gamma, \delta),
\end{aligned}
$$

where $\log$ denotes the principal branch of $\log$ and $m(\gamma, \delta)$ is an integer valued function dependent on $\Gamma(\gamma, \delta)$.

If $\Gamma$ does not contain $\infty$ and has algebraic endpoints $\gamma, \delta$ then the evaluation (4) already establishes the assertion. Similarly if $\Gamma$ is closed then taking $\gamma=\delta$ we see that $\log \left(\gamma-\alpha_{k}\right)-\log \left(\delta-\alpha_{k}\right)$ is always an integer multiple of $2 \pi i$ and thus a value of $\log 1$, whence (4) once again establishes the assertion and thus the theorem. 
If $\Gamma$ is a curve from $\gamma$ to $\infty$ then by letting $\delta$ tend to $\infty$ along $\Gamma$ we obtain (3) in the form

$$
\lim _{\delta \rightarrow \infty} \log \left\{\prod_{k=1}^{n}\left(\frac{\gamma-\alpha_{k}}{\delta-\alpha_{k}}\right)^{r k}\right\}+2 \pi i m(\gamma, \delta)
$$

$$
=\log \left\{\prod_{k=1}^{n}\left(\gamma-\alpha_{k}\right)^{r_{k}}\right\}+2 \pi i \lim _{\delta \rightarrow \infty} m(\gamma, \delta),
$$

since $\sum r_{k}=0$. By virtue of the existence of the integral (2) and the consequent existence of $(3), \lim _{\delta \rightarrow \infty} m(\gamma, \delta)$ exists, and $m(\gamma, \delta)=m(\gamma)$, an integer, for $\delta$ sufficiently large. But $2 \pi i m(\gamma)$ is a value of $\log 1$ whence (5) establishes the assertion in this case.

Similarly if $\Gamma$ is a curve from $\infty$ to $\infty$ we may let both $\gamma$ and $\delta$ tend to $\infty$ along $\Gamma$ in opposite directions from points between them. Then (3) becomes

$$
2 \pi i \lim _{\gamma, \delta \rightarrow \infty} m(\gamma, \delta)=2 \pi i m
$$

and as above, the double limit exists, so that (6) is a value of $\log 1$ and we again have the assertion.

I am indebted to the referee both for drawing my attention to the need for much of the detail of this section and for suggesting a suitable argument to deal with the case for $\Gamma$ containing $\infty$.

5. If $P(z)$ and $Q(z)$ satisfy the conditions of the corollary then already the partial fraction expansion (1) becomes

$$
\frac{P(z)}{Q(z)}=\sum_{k=1}^{n} \frac{r_{k}}{z-\alpha_{k}}
$$

and the corollary can be seen to follow immediately from the theorem.

6. Given the poles $\alpha_{k}$ and the residues $r_{k}$ at the poles of a rational function with algebraic coefficients it is not difficult to apply the criterion of the theorem in any specific case. For convenience of reference we mention explicitly the form of the criterion for $\Gamma$ lying along the real axis.

If $\gamma, \delta$ are algebraic and Log denotes the branch of $\log$ for which $\log 1=0$, then:

$\int_{\gamma}^{\delta}(P(x) / Q(x)) d x$ is algebraic if and only if

$$
\sum_{k=1}^{n} r_{k}\left\{\log \left(\delta-\alpha_{k}\right)-\log \left(\gamma-\alpha_{k}\right)\right\}=0 ;
$$


$\int_{\gamma}^{\infty}(P(x) / Q(x)) d x$ is algebraic if and only if

$$
\sum_{k=1}^{n} r_{k} \log \left(\gamma-\alpha_{k}\right)=0
$$

$\int_{-\infty}^{\infty}(P(x) / Q(x)) d x$ is algebraic if and only if it is zero.

\section{REFERENCES}

1. A. Baker, Linear forms in the logarithms of algebraic numbers. I-IV, Mathematika 13 (1966), 204-216; ibid. 14 (1967), 102-107, 220-228. MR 36 \#3732.

2. K. Mahler, Lectures on a theorem of $A$. Baker, Report of the Tenth Summer Research Institute of the Australian Mathematical Society (Hobart, 1970).

3. C. L. Siegel, Transcendental numbers, Ann. of Math. Studies, no. 16, Princeton Univ. Press, Princeton, N. J., 1949. MR 11, 330.

University of New South Wales, Sydney, Australia 